\title{
TIE POINTS EXTRACTION FOR SAR IMAGES BASED ON DIFFERENTIAL CONSTRAINTS
}

\author{
Xin Xiong, Guowang Jin, Qing Xu, Hongmin Zhang \\ Zhengzhou Institute of Surveying and Mapping, 450052 Middle Longhai Road, Zhengzhou, China \\ - (xiongxinhbhh, guowang_jin)@163.com
}

Commission VI, WG VI/4

KEY WORDS: Synthetic Aperture Radar (SAR), Tie Points, Matching, Extraction, Differential Constraints

\begin{abstract}
:
Automatically extracting tie points (TPs) on large-size synthetic aperture radar (SAR) images is still challenging because the efficiency and correct ratio of the image matching need to be improved. This paper proposes an automatic TPs extraction method based on differential constraints for large-size SAR images obtained from approximately parallel tracks, between which the relative geometric distortions are small in azimuth direction and large in range direction. Image pyramids are built firstly, and then corresponding layers of pyramids are matched from the top to the bottom. In the process, the similarity is measured by the normalized cross correlation (NCC) algorithm, which is calculated from a rectangular window with the long side parallel to the azimuth direction. False matches are removed by the differential constrained random sample consensus (DC-RANSAC) algorithm, which appends strong constraints in azimuth direction and weak constraints in range direction. Matching points in the lower pyramid images are predicted with the local bilinear transformation model in range direction. Experiments performed on ENVISAT ASAR and Chinese airborne SAR images validated the efficiency, correct ratio and accuracy of the proposed method.
\end{abstract}

\section{INTRODUCTION}

Synthetic Aperture Radar (SAR) is widely used in rapid terrain mapping of cloudy, foggy and rainy areas because of its full-day, all-weather imaging capabilities (Jin et al., 2015). In commonly used terrain mapping techniques with SAR images, radargrammetry technology is not limited by image coherence and has a practical application value in some specific scenes. However, as a fundamental step of radargrammetry, tie points (TPs) are mainly extracted by the operator of human-computer interaction, which seriously reduces the efficiency of SAR image terrain mapping (Jin et al., 2014).

Automatic extraction of TPs is usually based on image matching technology. However, it is challenging for SAR image matching due to the multiplicative speckle noise and the nonlinear geometric deformations. Broadly, researches on SAR image matching can generally be classified into two types. The first type makes some improvements in the scale-invariant feature transfor$\mathrm{m}$ (SIFT) operator (Fan et al., 2015). SIFT operator is capable of overcome the effects of geometric distortions greatly. Thus, these improvements focus on diminishing speckle influence. Typical methods include the SIFT-OCT method, which skips the features detected at the first octave of the scale space pyramid (Schwind et al., 2010); the BFSIFT method, which builds scale space by a bilateral filter (Wang et al., 2012); the ISIFT method, which transforms the noise model using logarithmic transformation (Suri et al., 2010); and the SAR-SIFT method, which defines a new gradient obtained from the ratio of exponentially weighted averages (ROEWA) algorithm (Dellinger et al., 2015, Zhu et al., 2016). The second type uses the edge-feature-based matching method. Although the extracted edges tend to be discrete and dubious on SAR image, they are more robust compared with the feature points. Common methods of this type include the method based on crossroad and road junction (Dell'Acqua et al., 2004); the method based on the strength and direction of the edge points (Chen et al., 2014, Chen and Chen, 2014); and the method based on edge features described by the distance and orientation to the center feature points (Zhang et al., 2015).

Although many of the aforementioned methods are effective to some extent, most of them are not validated by large-size SAR image experiments (Chen et al., 2007). As the image size increases, the efficiency of the two types of methods will be significantly reduced (Chen et al., 2008, Li et al., 2014). Moreover, the relative geometric distortions between large-size SAR images are not globally uniform. Therefore, the interest points may not be clearly distinguishable from one another, which results in mismatches.

To overcome these limitations, this paper mainly focuses on matching large-size SAR images obtained from approximately parallel tracks. This type of image is usually applied in radargrammetry and has obvious relative geometric distortion characteristics. The relative geometric distortions are small in azimuth direction and large in range direction. Taking into account the characteristics, this study proposes an automatic TPs extraction method based on differential constraints. Image pyramids are built firstly, and then corresponding layers of pyramids are matched from the top to the bottom. In the process, some improved technologies including normalized cross correlation (NCC) matching with rectangle window, false matches removal with differential constrained random sample consensus (DC-RANSAC) and matching point prediction with local modeling are presented to weaken the effects of geometry distortions. The efficiency, correct ratio and accuracy of the proposed method are confirmed by experiments on various types of SAR data obtained from platforms at different heights.

\section{PROPOSED METHOD}

The flow chart of the proposed method is shown in Fig. 1. The image pyramids are built for the reference and the sensed SAR 


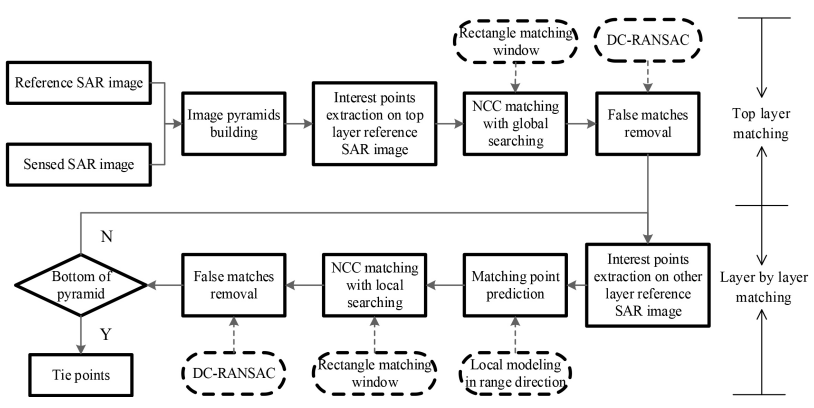

Figure 1. Flow chart of the proposed method.

image, respectively. In detail, the lower pyramid image is blurred by the Gaussian filter, and then $9(3 \times 3)$ pixels of the image are down sampled as 1 pixel of the upper pyramid image. Moreover, we use Moravec operator to extract interest points on each layer reference image since the proposed method only needs roughly uniformly distributed points on the reference image. Obviously, Moravec operator is simple and efficient, and can obtain the proper distribution and number of interest points with the local non-maximum suppressed. After the pyramids are built, the top layer is matched firstly, and then followed with the layer by layer matching.

Top layer matching: the interest points are extracted on the top layer reference SAR image. For each interest point, the corresponding candidate matching point is searched globally on the sensed image via the NCC with rectangle window. The principle of searching is to achieve the maximum correlation coefficient. After obtaining the candidate matches, the false matches are removed by the DC-RANSAC algorithm, and then obtaining the correct matches of the top layer.

Layer by layer matching: the interest points are extracted on the corresponding layer reference SAR image. The correct matches on the lower pyramid images are applied to establish the global bilinear transformation model in azimuth direction and the local bilinear transformation model in range direction, and then to predict the matching point of each interest point on the sensed image. The search window is centered at the predicted matching point, and the corresponding candidate matching point is searched locally in the search window via the NCC with rectangle window. After obtaining the candidate matches, the false matches are removed by the DC-RANSAC algorithm, and then obtaining the correct matches of the corresponding layer. Following the above steps, the pyramid images are matched layer by layer. Until the original images (pyramid bottom images) are matched, the TPs are obtained.

The key steps involved in the matching process include NCC with rectangle window, false matches removal with DC-RANSAC, matching point prediction with local modeling.

\subsection{NCC with Rectangle Window}

The normalized cross correlation measures the similarity between the interest points of the reference image and the image points of the sensed image by calculating the correlation coefficient of gray values in the neighborhood of two points (Ye et al., 2017). The correlation coefficient $\gamma$ ranges from -1 to +1 , and the closer $\gamma$ is to +1 , the more similar the two points will be. Thus, if the correlation coefficient $\gamma$ is locally maximum and larger than a certain threshold $\lambda$, the image point is the matching point of the corresponding interest point. The neighborhood of the points is called the matching window, which is generally rectangular. The length (azimuth length) and the width (range length) of the window are $m$ and $n$, respectively. As we known, the relative geometric distortions are small in azimuth direction and large in range direction for two SAR images. In the proposed method, the side of the matching window parallel to azimuth direction is larger than that parallel to range direction, that is $m>n$.

The correlation coefficient $\gamma$ is calculated as:

$$
\gamma=\frac{\sum_{i=-M}^{M} \sum_{j=-N}^{N}\left(f_{i+x, j+y}-\bar{f}\right)\left(g_{i+x^{\prime}, j+y^{\prime}}-\bar{g}\right)}{\sqrt{\sum_{i=-M}^{M} \sum_{j=-N}^{N}\left(f_{i+x, j+y}-\bar{f}\right)^{2} \sum_{i=-M}^{M} \sum_{j=-N}^{N}\left(g_{i+x^{\prime}, j+y^{\prime}}-\bar{g}\right)^{2}}}
$$

Where, $M=\operatorname{INT}(\mathrm{m} / 2), N=\operatorname{INT}(\mathrm{n} / 2)$, INT( $\bullet)$ round$\mathrm{s}$ the element of $\bullet$ to the nearest integer towards minus infinity. $f_{i+x, j+y}$ is the intensity value of the reference image at the image point $(i+x, j+y) \cdot g_{i+x^{\prime}, j+y^{\prime}}$ is the intensity value of the sensed image at the image point $\left(i+x^{\prime}, j+y^{\prime}\right) . \bar{f}$ and $\bar{g}$ are the means of the intensity values in matching windows for the two image, respectively.

\subsection{False Matches Removal with DC-RANSAC}

RANSAC algorithm is a general parameter estimation approach, which is commonly used in the false matches removal of image matching. The algorithm uses the candidate matches set that contains a large number of false matches to estimate the transformation model of the two images.

The bilinear transformation model can be used in RANSAC:

$$
\begin{gathered}
x_{2}=a_{0}+a_{1} x_{1}+a_{2} y_{1}+a_{3} x_{1} y_{1} \\
y_{2}=b_{0}+b_{1} x_{1}+b_{2} y_{1}+b_{3} x_{1} y_{1}
\end{gathered}
$$

(2) and (3) are the transformation model in range and azimuth direction, respectively. where, $x_{1}$ and $x_{2}$ are the range coordinates, $y_{1}$ and $y_{2}$ are the azimuth coordinates. $a_{i}$ and $b_{i}$ are the parameters of the transformation model.

If $\rho$ and $\varepsilon$ are the thresholds in range and azimuth direction, respectively, the criterions for determining the correct matches:

$$
\begin{gathered}
\left|a_{0}+a_{1} x_{1}+a_{2} y_{1}+a_{3} x_{1} y_{1}-x_{2}\right|=x_{\mathrm{off}} \leq \rho \\
\left|b_{0}+b_{1} x_{1}+b_{2} y_{1}+b_{3} x_{1} y_{1}-y_{2}\right| \leq \varepsilon
\end{gathered}
$$

Since the relative geometric distortions are small in azimuth direction and large in range direction for two images, the correct matches can accurately fit the bilinear transformation model in azimuth direction, but are difficult to fit the low order polynomial model (such as the bilinear transformation model) in range direction. Therefore, there is a coordinate offset $x_{\text {off }}$ between the calculated range coordinate and the correct range coordinate. The threshold $\varepsilon$ in (5) is a small value, and the threshold $\rho$ in (4) is related to the coordinate offset.

The RANSAC algorithm, with the bilinear transformation model (strong constraint model) in azimuth direction and the bilinear transformation model containing the coordinate offset (weak constraint model) in range direction, is called the DC-RANSAC algorithm. The algorithm is calculated as follows: 
1. Randomly select a sample (4 matches) in the candidate matches set to initialize the bilinear transform model according to (2) and (3).

2. Determine whether each match in the candidate matches set is correct, and form the correct matches set.

3. If the size of the correct matches set exceeds the threshold $\tau$, re-estimate the model parameters with the set data and end the algorithm.

4. Otherwise, select a new sample, and then repeat steps 1) to 3) $K$ times. Update the correct matches set using the largest correct matches set, and re-estimate the model parameters with the set data. Then end the algorithm.

5. Calculate the coordinate offset of each correct matches in range direction, and get the maximum coordinate offset of all the correct matches $x_{\text {offmax }}$.

\subsection{Matching Point Prediction with Local modeling}

The purpose of predicting the position of the matching point on the sensed image is to improve the matching efficiency. In this step, firstly map the correct matches of the upper pyramid to the lower pyramid. Then, establish the transformation model between the two images to complete the prediction.

The transformation model has been established when remove false matches, namely (2) and (3). However, there is a coordinate offset when predict matching points with (3). In this case, to search for the matching point in the neighborhood of the predicted point, the local search window for the NCC:

$$
\left\{-k x_{\text {offmax }}^{\prime} \leq x \leq k x_{\text {offmax }}^{\prime} ;-k \varepsilon / 2 \leq y \leq k \varepsilon / 2\right\}
$$

Where, $k$ is the multiple of the down sampling. $\varepsilon$ is the azimuth threshold of DC-RANSAC for the upper pyramid. $x_{\text {offmax }}^{\prime}$ is the maximum coordinate offset of all the candidate matches for the upper pyramid, $x_{\text {offmax }}^{\prime} \geq x_{\text {offmax }}$.

Since the range coordinates predicted by (2) are not accurate enough, the local search window represented by (6) is oversize, which reduces the matching efficiency. To overcome this problem, the local transformation model in range direction is needed. For each feature point $i, 4$ correct matches closest to it is searched on the lower reference image. These matches can estimate the parameters of a local bilinear transformation model, that is:

$$
x_{2}=a_{i 0}+a_{i 1} x_{1}+a_{i 2} y_{1}+a_{i 3} x_{1} y_{1}
$$

The global bilinear transformation model (2) and the local bilinear transformation model (7) can predict the matching point more accurately. Therefore, we set the local search window:

$$
\left\{-k x_{\text {offmax }} \leq x \leq k x_{\text {offmax }} ;-k \varepsilon / 2 \leq y \leq k \varepsilon / 2\right\}
$$

\section{EXPERIMENTS AND RESULTS}

\subsection{Test Datasets}

To evaluate applicability, three different types of SAR datasets obtained from platforms at different heights are tested. Dataset 1 is the spaceborne SAR data of the Henan Dengfeng mountain
Table 1. Parameters of three datasets.

\begin{tabular}{|c|c|c|c|}
\hline Parameter & Dataset 1 & Dataset 2 & Dataset 3 \\
\hline Type & Ground & Slant & Slant \\
\hline Band & C & X & Ka \\
\hline Aps (m) & 12.5 & 2.049 & 0.141 \\
\hline Rps (m) & 12.5 & 1.999 & 0.134 \\
\hline As (pixel) & 8868 & 3276 & 13440 \\
\hline Rs (pixel) & 8420 & 8192 & 8192 \\
\hline
\end{tabular}

Table 2. Parameters of layer by layer matching.

\begin{tabular}{|l|c|c|c|c|c|}
\hline Dataset & Layer & $\lambda$ & $\rho$ & $\varepsilon$ & Window \\
\hline \multirow{3}{*}{$\begin{array}{l}\text { Dataset } \\
1\end{array}$} & 1 & 0.5 & 20 & 3 & $7 \times 23$ \\
\cline { 2 - 6 } & 2 & 0.5 & $k x_{\text {offmax } 1}$ & 5 & $11 \times 35$ \\
\cline { 2 - 6 } & 3 & 0.5 & $k x_{\text {offmax } 2}$ & 5 & $17 \times 53$ \\
\cline { 2 - 6 } & 4 & 0.5 & $k x_{\text {offmax } 3}$ & 3 & $25 \times 79$ \\
\hline \multirow{3}{*}{\begin{tabular}{l} 
Dataset \\
\cline { 2 - 6 }
\end{tabular}} & 1 & 0.4 & 20 & 3 & $23 \times 43$ \\
\cline { 2 - 6 } & 2 & 0.4 & $k x_{\text {offmax } 1}$ & 5 & $35 \times 65$ \\
\cline { 2 - 6 } & 3 & 0.4 & $k x_{\text {offmax } 2}$ & 5 & $53 \times 97$ \\
\cline { 2 - 6 } Dataset & 4 & 0.4 & $k x_{\text {offmax } 3}$ & 3 & $79 \times 145$ \\
\cline { 2 - 6 } 3 & 1 & 0.4 & 20 & 3 & $7 \times 39$ \\
\cline { 2 - 6 } & 2 & 0.4 & $k x_{\text {offmax } 1}$ & 5 & $11 \times 59$ \\
\cline { 2 - 6 } & 3 & 0.4 & $k x_{\text {offmax } 2}$ & 5 & $17 \times 89$ \\
\cline { 2 - 6 } & 4 & 0.4 & $k x_{\text {offmax } 3}$ & 5 & $25 \times 133$ \\
\cline { 2 - 6 } & 5 & 0.4 & $k x_{\text {offmax } 4}$ & 3 & $37 \times 199$ \\
\hline
\end{tabular}

area obtained by European Space Agency ENVISAT ASAR system. Dataset 2 is the airborne SAR data of the Shanxi Weinan hilly area obtained by the airborne SAR System of Chinese Academy of Surveying and Mapping (CASM). Dataset 3 is the airborne SAR data of the Shanxi Yanliang hilly area obtained by the airborne SAR system of 23rd Institute, China Aerospace Science \& Industry Corp. The parameters of the three datasets (the reference and sensed images) including the image type, the wave band, the azimuth pixel spacing (Aps), the range pixel spacing (Rps), the azimuth size (As) and the range size (Rs) are shown in table 1.

\subsection{Experiments and Results}

Experiments on three datasets were designed. In the experiments, 4-layer pyramid was built for Dataset 1 and 2, and 5-layer pyramid was built for Dataset 3 . In addition, all the following experiments were carried out on a laptop with Intel Core i5 $2.40 \mathrm{GHz}$ processor and $1 \mathrm{~GB}$ RAM.

The threshold of NCC matching $\lambda$, the range and azimuth thresholds of DC-RANSAC ( $\rho$ and $\varepsilon$ ) as well as the size of matching window were shown in table 2. The range threshold of DCRANSAC was obtained by extending the maximum coordinate offset $x_{\text {offmax } j}$ by $k$ times ( $j$ was the layer number, $j=1,2,3,4$ ). The length and width of the each layer matching window were 1.5 times of the upper layer matching window. The size and shape of the top layer matching window was given directly here and would be discussed in section 3.4.

The matching results of the proposed method were analyzed quantitatively and shown in table 3 . The correctness of each match was identified by artificially comparing the corresponding points on the reference and the sensed SAR images. The evaluation criterion includes the total time $(\mathrm{Tt})$, the total time by parallel computation (Ttpc), the number of matches $(\mathrm{Nm})$, the number of correct matches by artificial comparison $(\mathrm{Ncmac})$ and the correct ratio by artificial comparison (Crac). The positions of the 
Table 3. Matching results for three datasets.

\begin{tabular}{|c|c|c|c|c|c|}
\hline Dataset & $\mathrm{Tt}(\mathrm{s})$ & $\mathrm{Ttpc}(\mathrm{s})$ & $\mathrm{Nm}$ & $\mathrm{Ncmac}$ & Crac (\%) \\
\hline Dataset 1 & 309 & 47 & 50 & 50 & 100.0 \\
\hline Dataset 2 & 358 & 39 & 38 & 38 & 100.0 \\
\hline Dataset 3 & 585 & 72 & 18 & 17 & 94.4 \\
\hline
\end{tabular}
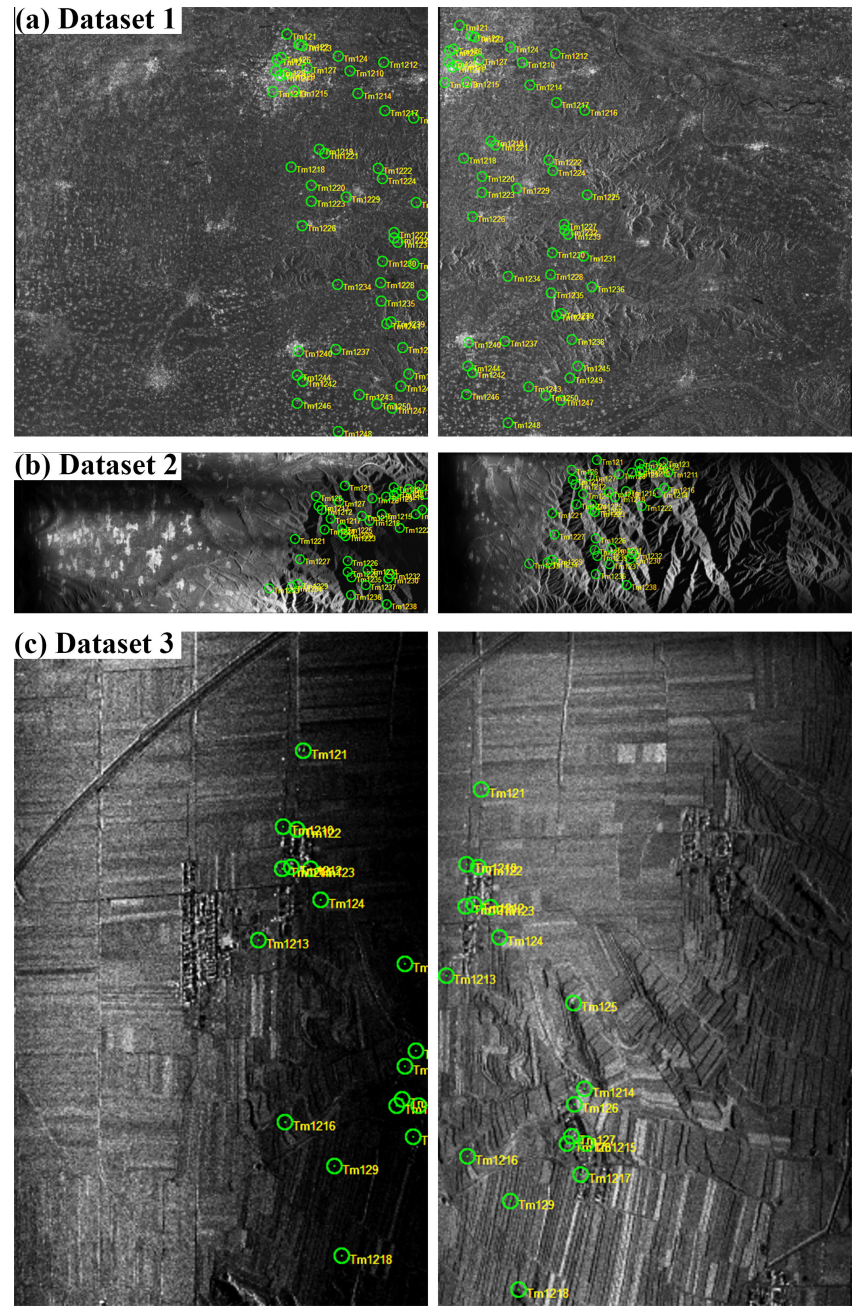

Figure 2. Matching results for three datasets.

matches (TPs) for the three datasets were shown in Fig. 2 and the randomly selected 6 matches (TPs) were shown in Fig 3.

In table 3, the correct ratio of Dataset 1 and 2 was $100.0 \%$, and of Dataset 3 was $94.4 \%$, where a false match appeared. Therefore, the correct ratio of the proposed method was high. However, the method could not ensure that all matches were correct, especially for Dataset 3, because the local texture of Dataset 3 was similar in some areas. After multi-thread parallel computing, the total time was reduced from over 5 minutes to about 1 minute indicating that the proposed method could achieve high computational efficiency through parallel computing.

In Fig. 2, the correct matches or TPs were uniformly distributed in the overlapping area because the interest points were extracted with the local non-maximum suppressed. Fig. 3 showed the details of 6 matches. The TP "Tm121" in Fig. 3 (c) was the false match.
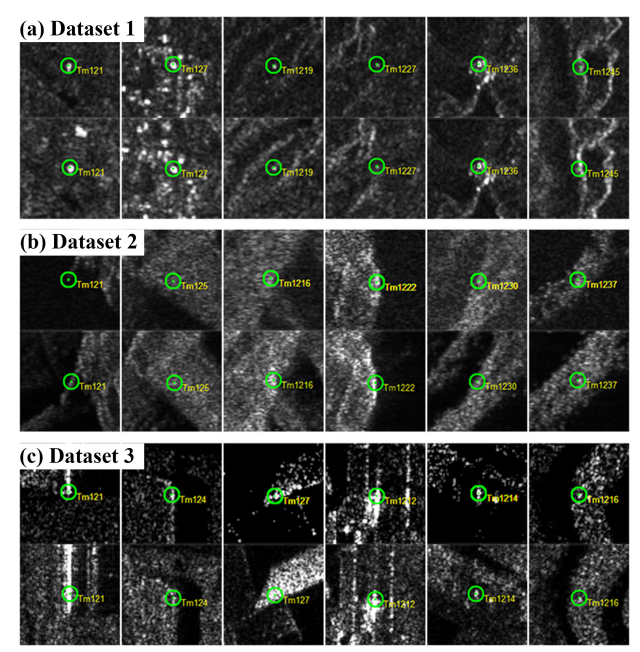

Figure 3. Six matching points on enlarged pictures for three datasets.

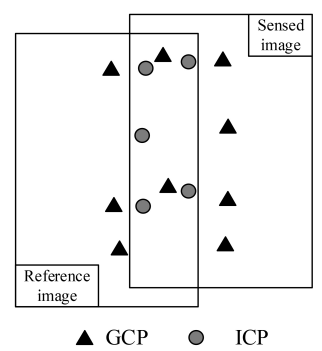

Figure 4. The distribution of GCPs and ICPs for Dataset 3.

Table 4. Errors and RMSE of ICPs for Dataset 3.

\begin{tabular}{|c|c|c|c|}
\hline Item & $X(\mathrm{~m})$ & $Y(\mathrm{~m})$ & $Z(\mathrm{~m})$ \\
\hline ICP1 & -0.127 & -0.086 & 0.134 \\
\hline ICP2 & 0.074 & -0.175 & -0.097 \\
\hline ICP3 & -0.056 & -0.127 & 0.054 \\
\hline ICP4 & 0.110 & 0.074 & -0.103 \\
\hline ICP5 & 0.049 & 0.343 & -0.020 \\
\hline RMSE & 0.089 & 0.188 & 0.091 \\
\hline
\end{tabular}

\subsection{Accuracy Analysis}

To objectively evaluate the accuracy of the extracted TPs, the stereoscopic orientation experiments were curried out for Dataset 3 . In the experiment, we constructed the error equations using the range-Doppler (r-D) model. r-D model is a rigorous geometric model widely used in geometric processing of SAR images (Schmitt et al., 2013). There were 5 ground control points (GCPs) on the reference image and 6 GCPs on the sensed image. 2 of them were conjugate GCPs, which were in overlapping areas between the image pair. 5 independent check points (ICPs), named ICP1, ICP2, ICP3, ICP4 and ICP5, were also collected. The ground coordinates of GCPs and ICPs were measured by $\mathrm{d}-$ ifferential GPS. The distribution of them was shown in Fig. 4. Subsequently, GCPs, ICPs and extracted TPs were used as input data for the stereoscopic orientation. The root mean square error (RMSE) of ICPs was computed to evaluate the accuracy of the stereoscopic orientation and it could also reflect the accuracy of TPs.

In table 4, the RMSE of ICPs was less than $0.2 \mathrm{~m}$, which was a smaller value. It indicated that the accuracy of the extracted TPs 


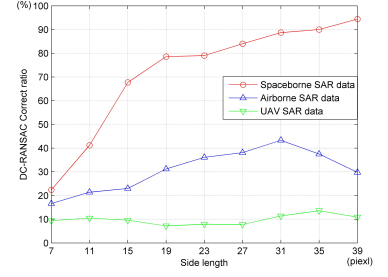

(a) DC-RANSAC correct ratio

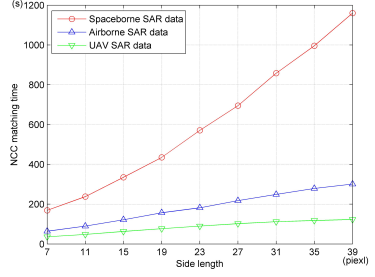

(b) NCC matching time
Figure 5. Top layer matching with square matching window.

was high, which can meet the needs of SAR image geometric processing.

\subsection{Discussion on Optimal Matching Window}

To determine the size and shape of the NCC matching window, the top layer matching experiments were performed under different size and shape of matching window. In the process, the DC-RANSAC correct ratio of matches $P$, which was the ratio of the correct matches to the candidate matches, was obtained after the false matches were removed.

Then, we could give the principle of selecting the optimal matching window (OMW). If $P$ was higher than $50 \%$, the matching window with the minimum matching time was OMW. If $P$ was lower than $50 \%$ and greater than $25 \%$, the matching window with the maximum correct ratio was OMW. If $P$ was lower than $25 \%$, there was no OMW.

The matching time included the time of NCC matching and DCRANSAC calculation. Compared to the NCC matching time $T$, the DC-RANSAC calculation time was expected to be very small, so the matching time here only included $T$.

The square matching windows with side lengths of $7,11,15,19$, $23,27,31,35$ and 39 as well as the rectangular matching windows with widths of $7,11,15,19,23$ and lengths of $11,15,19$, $23,27,31,35,39,43,47$ were designed for the top layer matching. The results were shown in Fig. 5 and 6.

From Fig. 5, we could find the optimal square matching windows for the three datasets. For Dataset $1, P$ could exceed $50 \%$. The optimal square matching window was $15 \times 15$ where the minimum matching time happened. For Dataset 2, $P$ did not exceed $50 \%$ but greater than $25 \%$. The optimal square matching window was $31 \times 31$ where $P$ reaches the maximum value of $40.2 \%$. For Dataset 2, $P$ was less than $25 \%$, so there was no optimal square matching window. From Fig. 6, we could also get the optimal rectangular matching windows for the three datasets, which was $7 \times 23,23 \times 43$ and $7 \times 39$, respectively, according to the principle of selecting OMW.

We could compare the optimal square matching window and the optimal rectangular matching window for the three datasets according to Fig. 5 and 6. For Dataset 1, when the matching window was $15 \times 15$ or $7 \times 23, P$ exceeded $50 \%$, and $T$ was 335 seconds and 262 seconds, respectively. Hence, the rectangular window had higher matching efficiency. For Dataset 2, when the matching window was $31 \times 31$ or $23 \times 43, P$ was $40.2 \%$ and $43.8 \%$, respectively, and $T$ was 249 seconds and 202 seconds, respectively. Hence, the rectangular window had higher matching accuracy and efficiency. For Dataset 3, there was no optimal square matching window, but there was an optimal rectangular
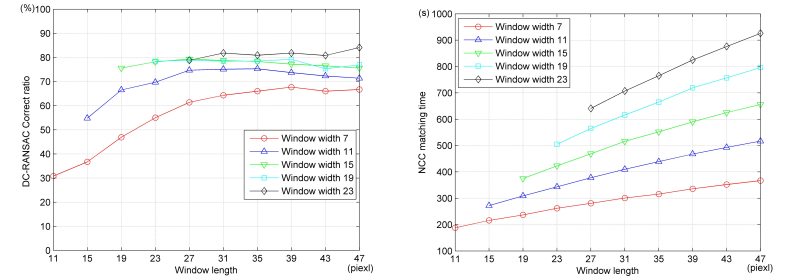

(a) DC-RANSAC correct ratio of (b) NCC matching time of Dataset 1 Dataset 1
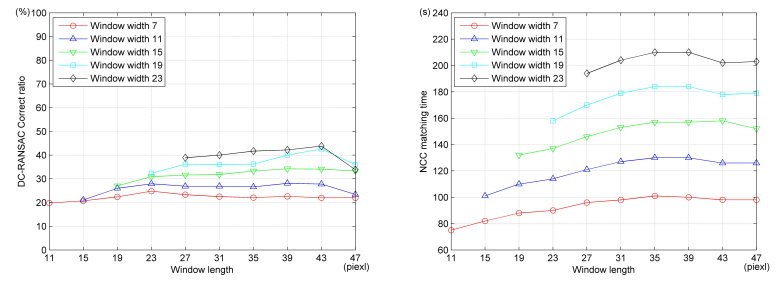

(c) DC-RANSAC correct ratio of (d) NCC matching time of Dataset 2 Dataset 2
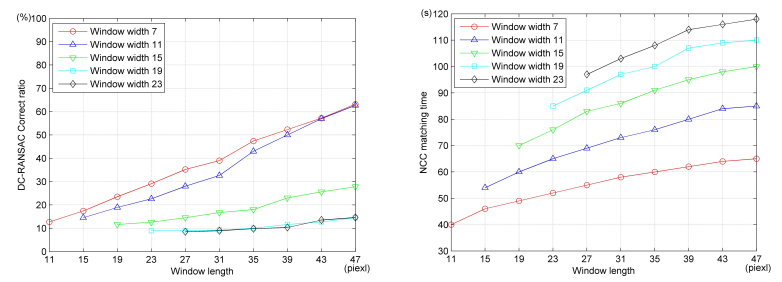

(e) DC-RANSAC correct ratio of (f) NCC matching time of Dataset 3 Dataset 3

Figure 6. Top layer matching with rectangular matching window.

matching window of $7 \times 39$. The above comparison showed that the rectangular window was more suitable for matching, and the improvements in the matching accuracy and efficiency were more obvious for Dataset 2 and 3. The reason was that the airborne SAR images used in the experiment were slant images. The relative geometric distortions in azimuth direction were larger than those in range direction.

Experiments on different size and shape of matching windows showed that the top layer matching window for the three datasets should be $7 \times 23,23 \times 43$ and $7 \times 39$, respectively.

\section{CONCLUSION}

This paper focuses on matching large-size SAR images obtained from approximately parallel tracks to extract TPs. The characteristics of the relative geometric distortion of such images are emphasized. In view of these characteristics, an automatic TPs extraction method based on normalized cross correlation matching with rectangle window, false matches removal with DC-RANSAC and matching point prediction with local modeling is proposed. The related experiments are performed on spaceborne and airborne, respectively. The proposed approach weakens the effects of geometry distortions. Hence, it can achieve high matching correct ratio and efficiency and accuracy under the optimal matching windows. In addition, it can extract TPs with high accuracy which can meet the needs of SAR image geometric processing. Also, it is suitable for various types of SAR data obtained from 
platforms at different heights. However, the DC-RANSAC algorithm in this paper does not completely remove false matches, and poor results will be obtained especially for local texture-like SAR images because there is only a strong constraint model in azimuth direction. This will be the subject of further work.

\section{ACKNOWLEDGEMENTS}

This work was supported by the National Natural Science Foundation of China (41071296, 41474010, 61401509). The authors would like to thank G.Chen et al. (Xi' an Research Institute of Surveying and Mapping) for their data.

\section{REFERENCES}

Chen, E., Li, Z.-Y., Tian, X. and Li, S.-M., 2008. Application of scale invariant feature transformation to sar imagery registration. Acta Automatica Sinica 34(8), pp. 861-868.

Chen, F., Wang, C. and Zhang, H., 2007. Automatic matching of high-resolution sar images. International Journal of Remote Sensing 28(16), pp. 3665-3678.

Chen, T. and Chen, L., 2014. A union matching method for sar images based on sift and edge strength. IEEE Journal of Selected Topics in Applied Earth Observations and Remote Sensing 7(12), pp. 4897-4906.

Chen, T., Chen, L. and Su, Y., 2014. A sar image registration method based on pixel migration of edge-point feature. IEEE Geoscience and Remote Sensing Letters 11(5), pp. 906-910.

Dell'Acqua, F., Gamba, P. and Lisini, G., 2004. Coregistration of multiangle fine spatial resolution sar images. IEEE Geoscience and Remote Sensing Letters 1(4), pp. 237-241.

Dellinger, F., Delon, J., Gousseau, Y., Michel, J. and Tupin, F., 2015. Sar-sift: a sift-like algorithm for sar images. IEEE Transactions on Geoscience and Remote Sensing 53(1), pp. 453-466.

Fan, J., Wu, Y., Wang, F., Zhang, Q., Liao, G. and Li, M., 2015. Sar image registration using phase congruency and nonlinear diffusion-based sift. IEEE Geoscience and Remote Sensing Letters 12(3), pp. 562-566.

Jin, G., Xu, Q. and Zhang, H., 2014. Synthetic aperture radar interferometry. National Defense Industry Press.

Jin, G., Zhang, H. and Xu, Q., 2015. Radargrammetry. Surveying and Mapping Press.

Li, B., Ming, D., Yan, W., Sun, X., Tian, T. and Tian, J., 2014. Image matching based on two-column histogram hashing and improved ransac. IEEE Geoscience and Remote Sensing Letters 11(8), pp. 1433-1437.

Schmitt, M., Maksymiuk, O., Magnard, C. and Stilla, U., 2013. Radargrammetric registration of airborne multi-aspect sar data of urban areas. ISPRS journal of photogrammetry and remote sensing 86, pp. 11-20.

Schwind, P., Suri, S., Reinartz, P. and Siebert, A., 2010. Applicability of the sift operator to geometric sar image registration. International Journal of Remote Sensing 31(8), pp. 1959-1980.

Suri, S., Schwind, P., Uhl, J. and Reinartz, P., 2010. Modifications in the sift operator for effective sar image matching. International Journal of Image and Data Fusion 1(3), pp. 243-256.

Wang, S., You, H. and Fu, K., 2012. Bfsift: A novel method to find feature matches for sar image registration. IEEE Geoscience and remote sensing letters 9(4), pp. 649-653.
Ye, Y., Shen, L., Hao, M., Wang, J. and Xu, Z., 2017. Robust optical-to-sar image matching based on shape properties. IEEE Geoscience and Remote Sensing Letters 14(4), pp. 564-568.

Zhang, H., Ni, W., Yan, W., Wu, J. and Li, S., 2015. Robust sar image registration based on edge matching and refined coherent point drift. IEEE Geoscience and Remote Sensing Letters 12(10), pp. 2115-2119.

Zhu, H., Ma, W., Hou, B. and Jiao, L., 2016. Sar image registration based on multifeature detection and arborescence network matching. IEEE Geoscience and Remote Sensing Letters 13(5), pp. 706-710. 